\title{
Cognitive Beamhopping for Spectral Coexistence of Multibeam Satellites
}

\author{
Shree Krishna SHARMA, Symeon CHATZINOTAS, Björn OTTERSTEN \\ SnT - securityandtrust.lu, University of Luxembourg, L-2721, Luxembourg \\ Tel: +352 4666445865, Fax: +352 4666445669, Email: shree.sharma@uni.lu
}

\begin{abstract}
The usable satellite spectrum is becoming scarce due to continuously increasing demand for broadcast and multimedia services. In this context, we consider the problem of enhancing spectral efficiency in multibeam satellite networks. The spectral coexistence scenario of two multibeam satellites over a common coverage area is studied with a primary satellite having larger beams and a secondary satellite having smaller beams. Furthermore, a novel cognitive beamhopping satellite system is proposed. The system performance of the proposed system is evaluated and compared with that of conventional multibeam and beamhopping systems in terms of system throughput. It is shown that the proposed system significantly enhances the spectral efficiency in comparison to other systems. Moreover, the scenario of the presence of multiple secondary users within an inactive primary beam is considered with power control on the secondary transmission. The system performance is evaluated by considering partial and full frequency sharing approaches. It is noted that the total spectral efficiency increases with the number of secondary users in the full frequency reuse approach.
\end{abstract}

Keywords: Beamhopping, Spectral Coexistence, Multibeam Satellites

\section{Introduction}

Next generation Satellite Communications (SatComs) systems are targeting enhanced throughput and higher spectral efficiency. To enhance the system capacity, satellite systems have moved from payloads generating a single beam to the multi-beam platform [1]. The frequency reuse concept can be applied in the multibeam satellite systems to provide higher capacity as in terrestrial cellular systems. However, the available spectrum resource is scarce and it is becoming congested as new broadband multimedia applications are introduced. In this context, exploring efficient spectrum sharing techniques to enhance spectral efficiency while guaranteeing Quality of Service (QoS) is a highly relevant and challenging problem. This has led to the concept of cognitive SatComs which exploits the opportunities for spectrum sharing between two satellite systems or between satellite and terrestrial systems [2].

The most common cognitive techniques in the literature can be categorized into Spectrum Sensing (SS) or interweave, underlay, overlay and database techniques. The spectrum coexistence literature is more mature in the terrestrial context but has received limited attention in the satellite context. Recent work exploiting spectrum sharing opportunities in the context of cognitive SatComs includes [2-6]. Furthermore, most of the current contributions in this context focus on hybrid coexistence scenario of satellite and terrestrial systems and only a few contributions address dual satellite coexistence scenario $[3,6,7]$.

In conventional multibeam systems, partial frequency reuse can be used to enhance the system capacity. However, it may be impractical to apply full frequency reuse due 
to excessive co-channel interference which is difficult to mitigate using existing interference mitigation techniques. A beamhopping satellite system can operate by using full frequency reuse over a certain beamhopping pattern [8]. The main difference between these systems is that in conventional multibeam systems, multiple beams within a cluster share available spectrum resource in the frequency domain whereas in the beamhopping satellite system, multiple beams within a cluster share available spectrum in the time domain. Several contributions exist in the literature in the context of beamhopping systems [8-11]. Since in a beamhopping system, only a single beam of a cluster is active during a particular time slot, there exists an opportunity to reuse the full frequency using smaller beams of another secondary satellite in the same time slot.

In this direction, we propose a cognitive beamhopping system with the objective of enhancing the system spectral efficiency while protecting the Primary Users (PUs). We consider a dual satellite spectral coexistence scenario of two multibeam satellites with a primary satellite having larger beams and a secondary satellite having smaller beams. The cognition is achieved by sharing the beamhopping pattern and the timing information of the primary multibeam system to the secondary multibeam system using a signalling link between their corresponding gateways. The timing information is exchanged to guarantee the proper synchronization of the primary and secondary transmissions. The primary multibeam system is considered to be an already deployed system and its performance should not be degraded beyond the prescribed threshold by the deployment of secondary satellite systems. The secondary satellite dynamically adapts its beampattern and transmit power ensuring the unobstructed operation of the primary system. In this context, the performance of the proposed cognitive beamhopping system is evaluated and compared with the performance of conventional multibeam and beamhopping satellite systems in terms of spectral efficiency (bits/sec/Hz). Furthermore, we consider full frequency reuse and frequency sharing among multiple

Secondary Users (SUs) within an inactive primary beam. Moreover, a power control technique is considered at the secondary satellite to protect the primary satellite terminals using a predetermined interference threshold.

The remainder of this paper is organized as follows: Section 2 reviews conventional satellite systems. Section 3 discusses the system model for the proposed cognitive beamhopping system. Section 4 presents the signal and channel model used for analysis. Section 5 provides the theoretical expressions for evaluating the performance of different techniques. Section 6 evaluates the system performance with the help of numerical results. Section 7 concludes the paper.

\section{Existing Satellite Systems}

\subsection{Conventional Multibeam System}

In conventional multibeam systems, the total available bandwidth $(W)$ in the forward link is divided into $K$ segments, where the parameter $K$ is the frequency reuse factor. Then the bandwidth allocated to the $i$-th user beam $\left(W_{i}\right)$ can be written as: $W_{i}=W / K$. The set of the beams which share the total bandwidth defines a beam cluster. The total gain in terms of frequency reuse obtained by using a multibeam satellite in comparison with a monobeam satellite depends on the number of clusters in that region which would be covered by a single beam of the monobeam satellite. The bandwidth allocated per beam can be written as: $W_{i}=N_{i} W_{\mathrm{c}}$, where $N_{i}$ is the number of carriers in the $i$ - 
th beam and $W_{\mathrm{c}}$ is the bandwidth of each carrier. As the value of $K$ decreases, the available bandwidth per beam increases but the co-channel interference also increases. Since the system capacity depends on both the available bandwith and the co-channel interference, the value of $K$ should be chosen in such a way that the maximum system capacity is acheived. The smallest possible value of $K$ in the conventional satellite systems is 3 [12].

\subsection{Flexible Multibeam System}

In comparison to the conventional systems, the flexible system uses a non-regular frequency reuse pattern and non-uniform power/carrier allocation. In practical situations, at least one carrier per beam is allocated i.e., $N_{i} \in\left\{1,2, \ldots, N_{\max }\right\}$, where $N_{\max }=N_{\mathrm{c}}-(K-1)$ and $N_{\mathrm{c}}=W / W_{\mathrm{c}}$ is the total number of available carriers. Let $N_{\mathrm{b}}$ be the total number of beams in the system, then the $N_{\mathrm{c}} \times N_{\mathrm{b}}$ bandwidth allocation matrix $\mathbf{C}$ can be defined as [11]:

$$
\mathbf{C}=\left[\begin{array}{cccc}
C_{11} & C_{12} & \ldots & C_{N_{\mathrm{b}} 1} \\
C_{12} & C_{22} & \ldots & C_{N_{\mathrm{b}} 2} \\
\vdots & \vdots & \ddots & \vdots \\
C_{1 N_{\mathrm{c}}} & C_{2 N_{\mathrm{c}}} & \ldots & C_{N_{\mathrm{b}} N_{\mathrm{c}}}
\end{array}\right]
$$

where $C_{i j}$ represents the $j$-th carrier allocated to $i$-th beam. The number of carriers allocated to $i$-th beam can be calculated as: $N_{i}=\sum_{j=1}^{N_{\mathrm{c}}} C_{i j}$, where $C_{i j} \in\{0,1\}$ indicates whether the $j$-th carrier is allocated to the $i$-th beam or not.

\subsection{Flexible Beamhopping Multibeam System}

In this system, a limited number of beams are simultaneously illuminated with a regular repetition pattern. This is referred to as a beamhopping technique. Such a technique helps to reduce the number of amplifiers on board as well as the power demands on the payloads [13]. This technique can be implemented with full frequency or partial frequency reuse. In case of full frequency reuse, a regular time window is periodically applied to the beamhopping system and the entire available bandwidth is allocated to each illuminated beam. The duration for each illuminated beam should be selected to satisfy the user transmission delay requirement. In case of partial frequency reuse, the total bandwidth is segmented and each beam can be illuminated with a fraction of $W$. Let $N_{\mathrm{t}}$ be the number of time slots in each time window, then the $N_{\mathrm{t}} \times N_{\mathrm{b}}$ beam illumination matrix $\mathbf{T}$ can be written as:

$$
\mathbf{T}=\left[\begin{array}{cccc}
T_{11} & T_{12} & \ldots & T_{N_{\mathrm{b}} 1} \\
T_{12} & T_{22} & \ldots & T_{N_{\mathrm{b}} 2} \\
\vdots & \vdots & \ddots & \vdots \\
T_{1 N_{\mathrm{t}}} & T_{2 N_{\mathrm{t}}} & \ldots & T_{N_{\mathrm{b}} N_{\mathrm{t}}}
\end{array}\right],
$$

where $T_{i j}$ indicates that the $j$-th time slot is allocated to the $i$-th beam. The total number of time slots allocated to the $i$-th beam can be written as: $N_{i, \mathrm{t}}=\sum_{j=1}^{N_{\mathrm{t}}} T_{i j}$, where $T_{i j} \in\{0,1\}$ indicates whether the $j$-th time slot is allocated to the $i$-th beam or not.

\section{Proposed Cognitive Beamhopping System}

We consider a dual satellite coexistence scenario as shown in Fig. 1. We consider both satellites to be multibeam satellites covering the same geographical region and 


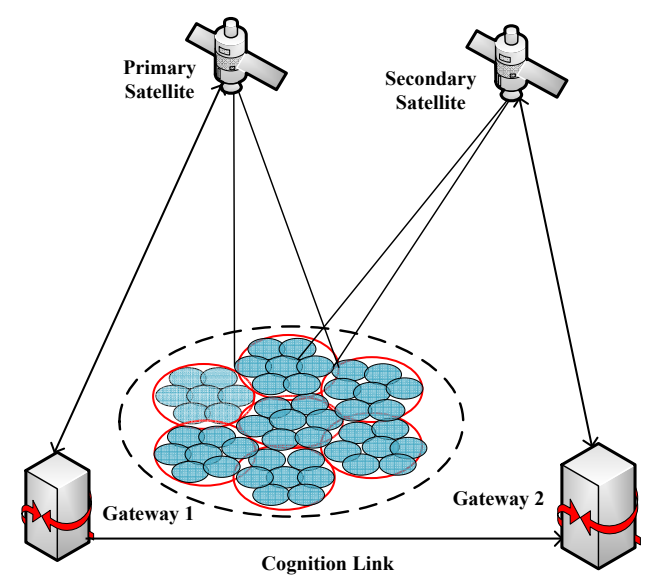

Figure 1: Spectral coexistence scenario of two multibeam satellites in the same geographic region

operating in the normal forward mode [2]. These satellites are connected to different gateways on Earth. Furthermore, we consider a primary satellite with larger beams and a secondary satellite with smaller beams. Consider the coverage area with larger primary beams and many spot-beams within each primary beam and these spot-beams are the beams of the secondary satellite. The importance of spot-beams is increasing due to their low peak gains and low contour levels resulting in smaller antenna aperture and lower hardware costs [14]. Furthermore, partitioning a beam into many sub-beams performs well towards the spot beam edge meeting the edge gain requirements.

Since the primary satellite only illuminates a small fraction of beams out of a large number of beams deployed under beamhopping systems, the rest of the beams remain idle at that time waiting for their transmission slots. If we could deploy a secondary satellite within the same spectrum in such a way that it has a beamhopping sequence different from that of the primary one and it does not produce harmful interference with the primary system, the overall system spectrum efficiency can be enhanced. The cognition is achieved by sharing the beamhopping pattern and the timing information of the primary satellite to the secondary satellite with the help of a signalling link between the gateways. Based on this knowledge of the beamhopping pattern, the secondary satellite's beamhopping pattern is designed so that it does not degrade the primary system's operation. Furthermore, the primary and secondary transmissions can be synchronized with the help of the exchanged timing information. In this context, different techniques such as an Exclusion Zone (EZ) principle [15] and a power control method can be applied for allowing the coexistence of primary and secondary systems. In this paper, we focus on beamhopping pattern planning for information exchange between two satellites and the power control aspect of interference management rather than the EZ method.

\section{Signal and Channel Model}

Let us consider a multibeam satellite system that employs a satellite antenna with $N_{\mathrm{b}}$ beams using a beamhopping pattern. At a particular time slot, only $M=N_{\mathrm{b}} / K$ beams are active transmitting independent information streams to $M$ fixed terminals located in different active main beams. During a particular time slot, each satellite terminal suffers from the interference from other co-channel beams. This multibeam satellite 
channel can be modeled with $M$ single user interfering links and is represented by an $M \times M$ channel matrix $\mathbf{H}$, each element of $\mathbf{H}, h_{i j}$ representing the channel coefficient from beam $j$ to user $i$. The beam gain of the $j$-th beam for the $i$-th user position can be denoted by $B_{i j}$ and can be written as [16]:

$$
B_{i j}=G_{\max } \cdot\left(\frac{J_{1}(u(i, j))}{2 u(i, j)}+36 \frac{J_{3}(u(i, j))^{2}}{u(i, j)^{3}}\right)^{2},
$$

where $u(i, j)=2.01723 \sin (\theta(i, j)) / \sin \left(\theta_{3 \mathrm{~dB}}\right), J_{m}$ is the first kind of Bessel's function of order $m$, and $G_{\max }$ is the maximum antenna gain, $\theta_{3 \mathrm{~dB}}$ is the 3 -dB angle and $\theta(i, j)$ represents the angular position of the $i$-th user from the $j$-th beam center with respect to the satellite. Let $N_{\mathrm{b} 1}$ be the total number of beams in the primary satellite and $M$ be the total number of users to be served by the network. Denote the received power at the output of the decoder of user $i$ by $P_{\mathrm{r}, i}$. It is related to the input power at beam $j$ as:

$$
P_{\mathrm{r}, i}=h_{i j} P_{\mathrm{t}, j},
$$

where $j=\left\{1, \ldots, N_{b 1}\right\}, i=\{1, \ldots, M\}, P_{\mathrm{t}, j}$ is the input power to beam $j$. Under clear sky conditions, this channel coefficient can be calculated as [17]:

$$
h_{i j}=\frac{B_{i j} G_{\mathrm{r}, i j}}{\left(4 \pi d_{i j}\right)^{2}}
$$

where $G_{\mathrm{r}, i j}$ is the gain of the $i$-th user antenna towards the $j$-th beam and can be considered to be constant as it does not show significant variation in time. The parameter $d_{i j}$ is the slant distance of the $i$-th user from the satellite which can be written as: $d_{i j}^{2}=r_{i, j}^{2}+D_{j}^{2}$, where $r_{i j}$ is the distance of $i$-th user position from the $j$-th beam centre position and $D_{j}$ is the height of the geostationary satellite from the $j$-th beam center position.

For each user $i$, the following condition should be satisfied to have a reliable link: $\gamma_{i} \geq \gamma_{\mathrm{th}}$, where $\gamma_{i}$ is the Signal to Interference plus Noise Ratio (SINR) of the $i$-th user which is defined for different systems in Section $5, \gamma_{\text {th }}$ is the minimum SINR required by the user to have the desired QoS. Therefore, the power allocated to each beam should be calculated in the following way.

$$
P_{\mathrm{t}, j}=\frac{\gamma_{\mathrm{th}} \cdot I_{\mathrm{cn}}}{\min _{i \in j}\left|h_{i j}\right|^{2}}
$$

where $I_{\mathrm{cn}}$ is a parameter including the noise and the interference from co-channel beams, the notation $i \in j$ means that $i$-th user is served by the $j$-th beam. The above equation represents the power allocated to the $j$-th beam under clear sky conditions.

\section{System Performance}

The following coexistence scenarios may be considered: (i) operating SUs only in the white region where no secondary interference is present, (ii) implementing power control in the secondary transmission, and (iii) by carrying out dynamic spectrum sensing and allocating idle bands to the SUs. In this work, we focus on power control of the secondary satellite to minimize interference towards the PUs. 


\subsection{Throughput Analysis}

In this section, we present the theoretical expressions used for performance evaluation of the different systems. We consider a conventional multibeam system and a beamhopping system with fixed carrier and power allocation.

\subsubsection{Conventional Multibeam System}

In this case, we consider the multibeam satellite coverage with frequency reuse factor of $K$. The dominant interference in this case is the co-channel interference from neighboring co-channel cells. The SINR of the $i$-th user is given by;

$$
\gamma_{\mathrm{CV}, i}=\frac{\left|h_{i i}\right|^{2} P_{\mathrm{t}}}{P_{\mathrm{t}} \sum_{j \in S_{\mathrm{P}}}\left|h_{i j}\right|^{2}+\sigma^{2}},
$$

where $\sigma^{2}$ denotes the noise power, $P_{\mathrm{t}}$ represents the transmitted power and $S_{\mathrm{P}}$ represents the set of co-channel beams. It can be noted that we include all possible co-channel cells in a given area and adjacent channel interference is not included in (7). The system throughput for this system can be written as:

$$
C_{\mathrm{CV}}=\frac{W}{K} \sum_{i=1}^{N_{b}} \log _{2}\left(1+\gamma_{\mathrm{CV}, i}\right)
$$

where $N_{\mathrm{b}}$ is the number of beams in the system.

\subsubsection{Beamhopping System}

In this case, we consider a beamhopping system with slot reuse factor of $K$. Each active beam uses full frequency instead of fractional frequency reuse as in the conventional multibeam systems. The SINR of the $i$-th user is given by;

$$
\gamma_{\mathrm{BH}, i}=\frac{\left|h_{i i}\right|^{2} P_{\mathrm{t}}}{P_{\mathrm{t}} \sum_{j \in S_{\mathrm{B}}}\left|h_{i j}\right|^{2}+\sigma^{2}},
$$

where $S_{\mathrm{B}}$ represents the set of beams which are active in a particular beamhopping slot. The system throughput for this system can be written as:

$$
C_{\mathrm{BH}}=W \sum_{i=1}^{N_{\mathrm{b}} / K} \log _{2}\left(1+\gamma_{\mathrm{BH}, i}\right)
$$

where $N_{b} / K$ represents the number of beams which are active per beam slot.

\subsubsection{Cognitive Beamhopping System}

Since only a certain fraction of total available beams are active in a particular time slot, we can explore the possibility of using those frequencies in the secondary satellite system in a secondary way. The primary system is a beamhopping system with larger beams with slot reuse factor of $K$. The secondary system can also be considered to be a beamhopping system with smaller beams and lower peak power. The total system throughput in this system can be expressed as:

$$
C_{\mathrm{CB}}=C_{\mathrm{P}}+C_{\mathrm{S}}=W\left(\sum_{i=1}^{N_{\mathrm{b}} / K} \log _{2}\left(1+\gamma_{\mathrm{CP}, i}\right)+\sum_{i=1}^{N_{\mathrm{s}}} \log _{2}\left(1+\gamma_{\mathrm{CS}, i}\right)\right)
$$


where $\gamma_{\mathrm{CP}, i}$ represents the SINR of the PU, $\gamma_{\mathrm{CS}, i}$ represents the SINR of the SU and $N_{s}$ denotes the number of active secondary beams. The expression for $\gamma_{\mathrm{CP}, i}$ can be written as:

$$
\gamma_{\mathrm{CP}, i}=\frac{\left|h_{\mathrm{P}, i i}\right|^{2} P_{\mathrm{pt}}}{P_{\mathrm{pt}} \sum_{j \in S_{\mathrm{P}}}\left|h_{\mathrm{p}, i j}\right|^{2}+P_{\mathrm{st}} \sum_{j \in S_{\mathrm{S}}}\left|h_{i j, \mathrm{~s}}\right|^{2}+\sigma^{2}},
$$

where $P_{\mathrm{pt}}$ is the transmit power of the primary system, $P_{\mathrm{st}}$ is the transmit power of the secondary system, $S_{\mathrm{S}}$ represents the set of secondary active beams in a particular slot, $h_{\mathrm{p}, i j}$ represents the channel gain of the $i$-th PU from the $j$-th primary beam and $h_{i j, \mathrm{~S}}$ represents the channel gain of $i$-th PU from the $j$-th secondary beam. Similarly, the expression for $\gamma_{\mathrm{CS}, i}$ can be written as:

$$
\gamma_{\mathrm{CS}, i}=\frac{\left|h_{\mathrm{S}, i i}\right|^{2} P_{\mathrm{st}}}{P_{\mathrm{pt}} \sum_{j \in S_{\mathrm{p}}}\left|h_{i j, \mathrm{P}}\right|^{2}+P_{\mathrm{st}} \sum_{j \in S_{\mathrm{S}}}\left|h_{\mathrm{S}, i j}\right|^{2}+\sigma^{2}},
$$

where $h_{i j, \mathrm{p}}$ is the channel gain of $i$-th SU from the $j$-th primary beam and $h_{\mathrm{s}, i j}$ is the channel gain of $i$-th SU from the $j$-th secondary beam.

\subsection{Power Control Method}

In this method, firstly, the aggregate interference from the secondary satellite beams to the PU is calculated and based on this interference level, the transmit power of the secondary satellite is adjusted to meet the interference threshold level of the PU. Let $I_{\mathrm{T}}$ be the interference threshold level of the PU to have sufficient protection and $I_{\mathrm{AGG}}$ be the aggregate interference from secondary beams to the PU. Then the expression for $I_{\mathrm{AGG}}$ at a particular slot can be written as: $I_{\mathrm{AGG}}=P_{\mathrm{st}} \sum_{j \in S_{\mathrm{S}}}\left|h_{i j, \mathrm{~s}}\right|^{2}$. The transmit power of the secondary satellite can be adjusted in the following way to guarantee sufficient protection for the PU.

$$
P_{\mathrm{st}}=\frac{I_{\mathrm{T}}}{\sum_{j \in S_{\mathrm{S}}}\left|h_{i j, \mathrm{~s}}\right|^{2}},
$$

It can be noted that as the number of SUs within an primary inactive beam increases, the denominator term of the above equation increases and the secondary satellite has to reduce its transmission power.

\section{Numerical Results}

We consider the following three systems for comparison: (i) conventional multibeam system (Section 5.1.1), (ii) beamhopping system (Section 5.1.2), and (iii) proposed cognitive beamhopping system (Section 5.1.3). The simulation parameters are presented in Table I. In the proposed cognitive beamhopping system, each beam of the primary system includes 7 sub-beams which are served by the secondary satellite. Figure 2 shows the spectral efficiency (bits/sec/Hz) versus SNR for different systems for $K=3, N_{\mathrm{b}}=$ 19 and $P_{\mathrm{st}}=P_{\mathrm{pt}}=10 \mathrm{dBW}$. From the figure, it can be noted that the spectral efficiency for the beamhopping system is slightly greater than for the conventional multibeam system. Furthermore, it can be noted that the primary only spectral efficiency slightly decreases at higher values of SNR in the presence of secondary system whereas total spectral efficiency of the cognitive beamhopping system increases. The increase in secondary throughput in comparison to primary throughput in Fig. 2 comes from the fact that there are more number of active secondary beams (smaller) with the same transmit power during a particular beamhopping slot. 
Table 1: Simulation Parameters

\begin{tabular}{l|c|c|} 
Parameter & Symbol & Value \\
\hline Orbit & & GEO \\
Frequency band & $R$ & Ka $(20 \mathrm{GHz})$ \\
Coverage area radius & $I_{0}$ & $-123 \mathrm{dBW}$ \\
Interference tolerance threshold & $K$ & 3,7 \\
Frequency/slot reuse factor & $N_{\mathrm{b}}$ & 19,37 \\
Number of primary Beams & $N_{s}$ & 7 \\
Number of secondary beams per $N_{\mathrm{b}}$ & $\theta_{3 \mathrm{~dB} 1}$ & $0.1325^{\circ}$ \\
3 dB beamwidth of primary & $\theta_{3 \mathrm{~dB} 2}$ & $0.05^{\circ}$ \\
$3 \mathrm{~dB}$ beamwidth of secondary & $G_{\mathrm{t}}$ & $52 \mathrm{dBi}$ \\
Max satellite antenna gain & $G_{\mathrm{r}}$ & $40 \mathrm{dBi}$ \\
Max user antenna gain & $F L$ & $210 \mathrm{~dB}$ \\
Free space path loss & $N_{o}$ & $-117 \mathrm{dBW}$ \\
Noise Power @ 500 MHz & & \\
\hline
\end{tabular}

To calculate the number of SUs which can be served in the switched off region of the primary beams, simulations were carried out by considering the presence of multiple SUs within each inactive primary beam. Figure 3(a) shows spectral efficiency versus number of SUs for the cognitive beamhopping system. In this simulation settings, the SUs were placed at the center of sub-beams in each inactive primary beam and the number of SUs was varied from 1 to 7 . The full frequency reuse is considered for both primary and secondary beamhopping systems. For the results in Fig. 3, the parameters considered were $K=7, N_{\mathrm{b}}=37, P_{\mathrm{pt}}=10 \mathrm{dBW}$. The interference tolerance threshold of each PU was considered to be $-123 \mathrm{dBW}^{1}$ and based on this interference threshold, power of the secondary satellite was calculated for the considered number of users. It can be noted that the spectral efficiency increases with the number of users and it almost saturates while increasing the user number from 6 to 7 . Furthermore, it can be noted that the transmit power of the secondary satellite needs to be decreased as number of SUs increases to protect the primary rate with a predetermined threshold. Moreover, the simulations were carried for the cases of $\left(K=3, N_{\mathrm{b}}=37\right),\left(K=7, N_{\mathrm{b}}=19\right)$ and $\left(K=3, N_{\mathrm{b}}=19\right)^{2}$. It has been noted that for a specific value of $N_{b}$, the total cognitive beamhopping throughput was higher for $K=7$ case for all the considered number of users but the primary throughput was higher for $K=3$ case. Furthermore, the primary protection rate was found to be slightly less in $K=3$ case than in $K=7$ case provided the same interference threshold limit for each PU.

To evaluate the performance of proposed cognitive beamhopping system in the presence of multiple users with frequency sharing, simulations were carried out by sharing the total spectrum among the SUs within the same inactive primary beam. In this case, each SU uses only a fraction of the spectrum resource and this fraction depends on the number of SUs. Figure 3(b) shows the spectral efficiency versus number of users for this scenario. It can be noted that the total throughput of the cognitive system decreases as the number of SUs increases for this scenario. Furthermore, the power of the secondary transmission has to be adjusted to meet the interference threshold limit of the PU as reflected in Fig. 3(b). From the comparison of above two approaches, it can be concluded that the spectral efficiency increases with the number of users in full frequency reuse

\footnotetext{
${ }^{1}$ This value was chosen to ensure that the aggregated interference to noise ratio does not exceed $-6 \mathrm{~dB}$.

${ }^{2}$ These results are not included in this paper due to space limitation.
} 


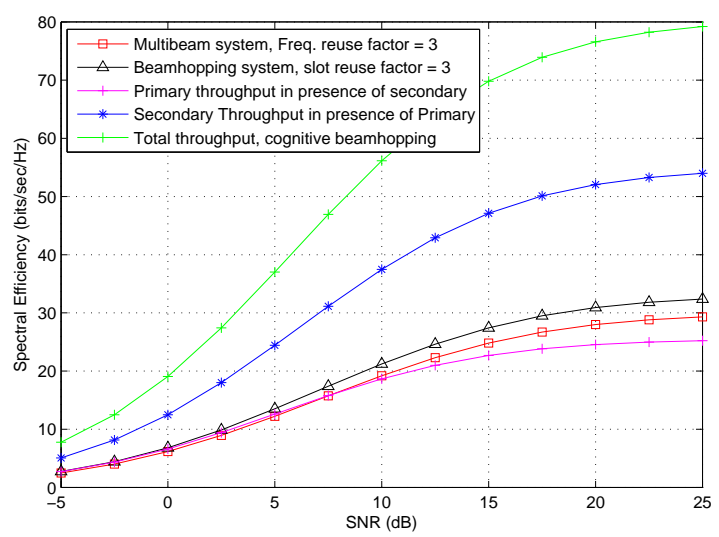

Figure 2: Throughput comparison of different systems $\left(K=3, N_{\mathrm{b}}=19, P_{\mathrm{st}}=P_{\mathrm{pt}}=10 \mathrm{dBW}\right)$

approach while it decreases with the number of users in frequency sharing approach. Furthermore, frequency sharing among users is not that much suitable from practical perspectives. Therefore, in practical scenarios, the cognitive beamhopping system can achieve significant enhancement in total throughput than the primary only system by using full frequency reuse approach in the secondary system.

\section{Conclusions}

In this work, a cognitive beamhopping technique has been proposed for the spectral coexistence scenario of two multibeam satellites. With the help of prior knowledge of the primary beamhopping pattern and the synchronization, the secondary satellite beamhopping pattern can be designed to ensure sufficient protection to the primary system. The performance of the proposed cognitive beamhopping system has been evaluated and compared with the conventional multibeam and beamhopping systems. It can be concluded that cognitive beamhopping system significantly improves the spectral efficiency over other techniques. Furthermore, based on the comparison of the two approaches, it can be concluded that the spectral efficiency increases with the number of users in the full frequency reuse approach and decreases with the number of users in the frequency sharing approach. Investigating different regions for the primary beams based on EZ principle and the application of dynamic spectrum sensing for exploiting temporal spectral holes are topics for future work.

\section{Acknowledgement}

This work was supported by the National Research Fund, Luxembourg under AFR (Aids Training-Research) grant for PhD project (Reference 3069102) on "Spectrum Sensing, Resource Allocation and Resource Management Strategies for Satellite Cognitive Communications", under the CORE project "CO2SAT: Cooperative and Cognitive Architectures for Satellite Networks".

\section{References}

[1] R. Alegre-Godoy, N. Alagha, and M. Vazquez-Castro, "Offered capacity optimization mechanisms for multi-beam satellite systems,” in Proc. IEEE Int. Conf. Commun., pp. 3180 -3184, June 2012.

[2] S. K. Sharma, S. Chatzinotas, and B. Ottersten, "Satellite cognitive communications: Interference modeling and techniques selection,” in Proc. 6th ASMS/SPSC Conf., Sept. 2012. 


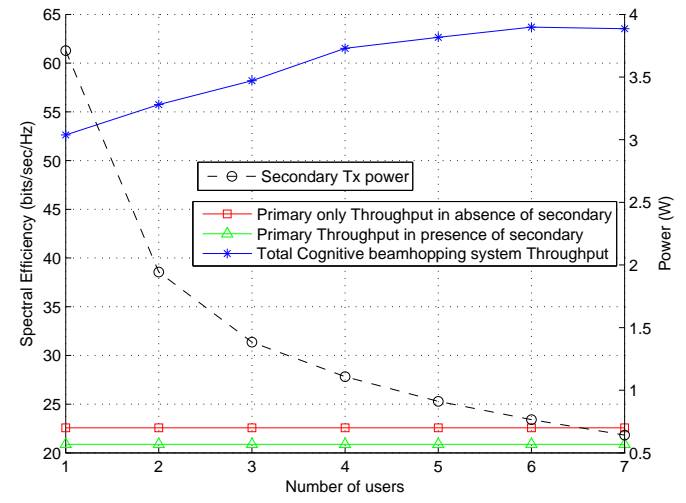

(a) With full frequency reuse

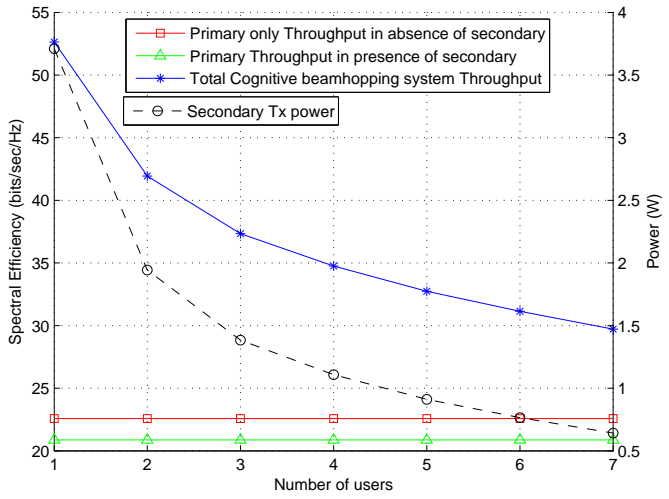

(b) With frequency sharing

Figure 3: Spectral efficiency versus number of users $\left(K=7, N_{\mathrm{b}}=37, P_{\mathrm{pt}}=10 \mathrm{dBW}\right)$

[3] S. K. Sharma, S. Chatzinotas, and B. Ottersten, "Exploiting polarization for spectrum sensing in cognitive SatComs," in Proc. 7th Int. Conf. CROWNCOM, June 2012.

[4] S. K. Sharma, S. Chatzinotas, and B. Ottersten, "Spectrum sensing in dual polarized fading channels for cognitive SatComs," in Proc. IEEE GLOBECOM, Dec. 2012.

[5] S. Kandeepan, L. De Nardis, M.-G. Di Benedetto, A. Guidotti, and G. Corazza, "Cognitive satellite terrestrial radios," in Proc. IEEE GLOBECOM, pp. 1 -6, Dec. 2010.

[6] Y. H. Yun and J. H. Cho, "An orthogonal cognitive radio for a satellite communication link," in Proc. IEEE 20th Int. Symp. on Personal, Indoor and Mobile Radio Commun., pp. 3154 -3158, Sept. 2009.

[7] S. K. Sharma, S. Chatzinotas, and B. Ottersten, "Interference alignment for spectral coexistence of heterogeneous networks," EURASIP J. Wireless Commun. and Networking, vol. 46, 2013.

[8] J. Anzalchi, A. Couchman, P. Gabellini, G. Gallinaro, L. D'Agristina, N. Alagha, and P. Angeletti, "Beam hopping in multi-beam broadband satellite systems: System simulation and performance comparison with non-hopped systems," in Proc. 5th ASMS conf. and the 11th SPSC workshop, pp. 248 -255, Sept. 2010.

[9] A. Mokhtar and M. Azizoglu, "On the downlink throughput of a broadband LEO satellite network with hopping beams," IEEE Commun. Letters, vol. 4, pp. 390 -393, Dec. 2000.

[10] N. Fonseca and J. Sombrin, "Multi-beam reflector antenna system combining beam hopping and size reduction of effectively used spots," IEEE Antennas and Propag. Mag., vol. 54, pp. 88 -99, April 2012.

[11] L. Jiang, Multi-beam Satellite Resource Optimization for Beam Hopping Transmission. Phd thesis, Department of Telecommun. and systems Engineering, Universitat Autonoma de Barselona, 2010.

[12] V. Boussemart, M. Berioli, F. Rossetto, and M. Joham, "On the achievable rates for the return-link of multi-beam satellite systems using successive interference cancellation," in Proc. Military Commun. Conf., pp. $217-223$, Nov. 2011.

[13] ISI, "Integral satcom initiative european technology platform strategic research and innovation agendaedition 2011.” Seventh Framework Programme (FP), 2011. Available online: www.isi-initiative.org.

[14] O. Kilic, "Interference analysis for spot beam partitioning in cellular satellite communication systems," in Proc. IEEE Antennas Propagat. Society Int. Symp., pp. 1 -4, July 2008.

[15] M. Vu, N. Devroye, and V. Tarokh, "On the primary exclusive region of cognitive networks," IEEE Trans. Wireless Commun., vol. 8, pp. 3380 -3385, July 2009.

[16] M. Diaz, N. Courville, C. Mosquera, G. Liva, and G. Corazza, "Non-linear interference mitigation for broadband multimedia satellite systems," in Proc. IWSSC, pp. 61 -65, Sept. 2007.

[17] A. Destounis and A. Panagopoulos, "Dynamic power allocation for broadband multi-beam satellite communication networks," IEEE Commun. Letters, vol. 15, pp. 380 -382, April 2011. 